Referencia para citar este artículo: Posada-Escobar, J. J., Briceño-Alvarado, P del P. \& Munar-Moreno, Y. A. (2017). Análisis de experiencias y dispositivos en construcción de paz desde una perspectiva intergeneracional-intercultural. Revista Latinoamericana de Ciencias Sociales, Niñez y Juventud, 15(1), pp. 505-517.

\title{
Análisis de experiencias y dispositivos en construcción de paz desde una perspectiva intergeneracional-intercultural*
}

\author{
Jorge Jatro Posada-EscobaR ** \\ Profesor e investigador de la Universidad Pedagógica Nacional, Colombia. \\ Patricia del Pilar Briceño-Alvarado ${ }^{* * *}$ \\ Profesora e investigadora del Centro Internacional de Educación y Desarrollo Humano-Cinde, Colombia. \\ YUDI ASTRID MUNAR-MORENO ${ }^{* * * * *}$ \\ Profesora e investigadora de la Universidad Pedagógica Nacional, Colombia.
}

\section{Artículo recibido en abril 22 de 2016; artículo aceptado en mayo 31 de 2016 (Eds.)}

- Resumen (analítico): Este artículo aborda las categorías intergeneracionalidad e interculturalidad como ejes de análisis de las experiencias y del dispositivo desarrollado por el Secretariado Nacional de Pastoral Social (SNPS), para propiciar la configuración de subjetividades políticas de paz y la transformación social. De igual forma, se explora el concepto de dispositivo que permite identificar los elementos de la propuesta metodológica de "Intervención para la transformación social" del SNPS.

Palabras clave autores: Experiencias de paz, interculturalidad-intergeneracionalidad, dispositivo, metodología de construcción de paz.

Palabras clave descriptores: Paz (Tesauro de Ciencias Sociales de la Unesco).

\section{Analysis of experiences and devices in peace-building from an intergenerational-intercultural perspective}

- $\quad$ Abstract(analytical): This article discusses the categories intergenerational and intercultural as axes of analysis of experiences and the device developed by the National Secretariat of Social Pastoral (SNPS), to promote political subjectivities configuration peace and social transformation.

Artículo corto que presenta resultados parciales de la investigación: Las condiciones y modos de construcción de subjetividades políticas para la paz, en jóvenes de organizaciones interétnicas y feministas desde una perspectiva intergeneracional, adelantada por el equipo del que hacen parte los autores, con el Secretariado Nacional de Pastoral Social mediante convenio del 12 de agosto de 2013. El proyecto hace parte del Programa cofinanciado por Colciencias. Código 12 35-543 31810. Contrato: 959-2012. Área: Ciencias Sociales, Interdisciplinaria. Subárea: Otras Ciencias Sociales. Fecha de inicio. marzo 2013 fecha de finalización: marzo 2016.

** Licenciado en Ciencias Sociales, Universidad Nacional. Magister en Desarrollo Educativo y Social Universidad Pedagógica-Cinde. Doctor en Filosofía y Educación, Uned, Madrid. Profesor e investigador, Universidad Pedagógica Nacional. Correo electrónico: jposada@pedagogica.edu.co

*** Psicóloga. Pontificia Universidad Javeriana. Magister en Desarrollo Educativo y Social, Universidad Pedagógica Nacional-Cinde. Profesora e investigadora. Correo electrónico: pbriceno@cinde.org.co

**** Licenciada en Psicología y Pedagogía, Universidad Pedagógica Nacional. Especialista en Programas de Educación y Alfabetización de personas jóvenes y adultas, Universidad Autónoma de Barcelona. Magister en Educación, Universidad Pedagógica Nacional. Candidata a doctora en Educación, Universidad de la Salle, Costa Rica. Profesora e investigadora de la Universidad Pedagógica Nacional. Correo electrónico: ymunar@ pedagogica.edu.co 
Similarly, the concept of device that identifies the elements of the methodology of "Intervention for social transformation" of SNPS is explored.

Authors key words: Experiences of peace, intercultural-intergenerationality, dispositive, method of peacebuilding.

Key words: Peace (Unesco Social Science Thesaurus).

\section{Análise de experiências e dispositivos em construção de paz desde uma perspectiva intergeneracional-intercultural}

- $\quad$ Resumo (analítico): Este artigo discute o intergeracional e intercultural categorias como eixos de análise de experiências eo dispositivo desenvolvido pela Secretaria Nacional de Pastoral Social (SNPS), para promover a paz configuração de subjetividades política e transformação social. Da mesma forma , o conceito de dispositivo que identifica os elementos da metodologia de "intervenção para a transformação social " de SNPS é explorado .

Palavras-chave autores: Experiências de paz, interculturalidade-intergeracionalidade, dispositivo, metodologia de construção de paz.

Palavras-chave descritores: Paz (Thesaurus de Ciências Sociais da Unesco).

-1. Introducción. -2. Metodología de la investigación. -3. IntergeneracionalidadInterculturalidad. -4. La estrategia de intervención del SNPS como dispositivo para la construcción de la Paz. -5. Lo intercultural e intergeneracional en las ILP y GSP. -Lista de referencias.

\section{Introducción}

La investigación adelantada es una sistematización de experiencias, que se realiza en Convenio con el Secretariado Nacional de Pastoral Social (SNPS) de la Iglesia Católica ${ }^{1}$.

En el año 2009, la sección de Vida, Justicia y Paz del SNPS formula el Proyecto "Construcción de Paz en cinco regiones y 15 Jurisdicciones Eclesiásticas de Colombia, con el que se pretende apalancar a las comunidades que, en medio de condiciones de violencia e inequidad, se han resistido a emplear la misma lógica y han propuesto salidas constructivas a su situación (Secretariado Nacional de Pastoral Social, s. f., p. 4).

El supuesto que subyace a este proyecto de la Iglesia es que:

... si las comunidades logran afirmar su rol como actores sociales transformadores en articulación con otros actores locales, regionales, nacionales e internacionales,

1 Institución creada por la Conferencia Episcopal Colombiana en 1977 con el propósito de orientar los esfuerzos de diversos organismos y movimientos eclesiales "por el desarrollo humano integral, sostenible y solidario a la luz del Evangelio y con los principios que brinda la Doctrina Social de la Iglesia" (Pastoral Social-Cáritas Colombia, 2015, párrafo 2). ello podría representar en el contexto colombiano un espacio con sólidas posibilidades de impactar positivamente el contexto Histórico de injusticia, inequidad y violencia (SNPS, s. f., p. 4).

Es así, como las Iniciativas Locales de Paz (ILP) y los Grupos de Sembradores de Paz (GSP), surgen como una alternativa de la Iglesia Católica al conflicto armado que ha hecho presencia en el país durante muchas décadas, con el propósito de fortalecer la democracia participativa y plural. Se entienden por ILP, ... todos aquellos procesos que a largo plazo buscan la construcción de sociedades y comunidades pacíficas y estables, abordando causas estructurales de los conflictos violentos, mediante la transformación de relaciones en el nivel local mediante el desarrollo de algún tipo de iniciativa comunitaria que cree espacios de diálogo, concertación, negociación, inclusión social, desarrollo, participación e incidencia para la búsqueda de mejores condiciones de vida. (SNPS, s. f., p. 5).

Para acompañar y apoyar estos procesos comunitarios, en el año 2011, el SNPS diseñó una Estrategia de Intervención Social para la 
Construcción de Paz. Esta, es fruto del trabajo histórico realizado con las comunidades en el país, a través de diversos programas orientados desde la doctrina social de la Iglesia con opción por los pobres y de la reflexión acerca de las maneras como se ha llevado a cabo la Pastoral Social.

Ahora bien, esta sistematización busca analizar estas experiencias del SNPS desde una perspectiva intergeneracional-intercultural, y su estrategia de intervención para la transformación social, como dispositivo.

En este artículo, en primera instancia se explica la metodología de la investigación $\mathrm{y}$ se hace una breve descripción de las experiencias sistematizadas, posteriormente, se exponen las categorías intergeneracionalidad e interculturalidad como ejes de análisis de las experiencias y del dispositivo para la construcción de subjetividades de paz.

\section{Metodología de la investigación}

Esta investigación se realizó por medio de la sistematización de experiencias en seis ILP y en dos GSP promovidas por el SNPS en Bogotá, Cundinamarca, Boyacá, Caldas, Cauca, Huila y Putumayo.

La sistematización de experiencias, como modalidad de investigación social, se interesa por producir conocimiento en y desde las prácticas sociales. Se propone reconocer los sentidos otorgados por los sujetos actores de estas prácticas. Esta lógica coloca de presente la importancia que tiene para esta modalidad investigativa, la visibilización de los saberes, que como construcciones de lenguaje y de cultura, fundamentan y orientan las prácticas sociales. Son estos saberes, más allá de las descripciones de las prácticas y experiencias, los que constituyen el núcleo central de la sistematización de experiencias (Ramírez, 2009).

Descifrar estos saberes en la trama de las prácticas sociales es poner en evidencia las lógicas de su constitución, las relaciones entre actores y su lugar en los escenarios y temporalidades que las contextualizan y les dan historicidad. Es el ejercicio de comprensión crítica que hacen los sujetos de sus prácticas, de su lugar en ellas.

Se consideró importante realizar esta sistematización desde un enfoque hermenéutico que desarrolla y aprovecha la narración, ya que esta se constituye en una forma primordial de expresión de las subjetividades de las personas.

De ahí que, en esta investigación un elemento muy importante son las narrativas de los actores, las experiencias contadas como historias, en ellas se puede escudriñar los sentidos de las acciones de paz que han vivido las organizaciones y las personas de los GSP e ILP.

Por medio de las narraciones las personas construyen su identidad, son un elemento metodológico que fortalece o expresa la memoria, a través de ella los actores dan significado, interpretan sus acciones y sus experiencias.

Para esta sistematización se siguieron las fases metodológicas sugeridas por Cendales, Mariño y Posada (2004). Estas fases se dieron en gran parte de forma simultánea.

En la fase de preparación, se conformó el equipo de investigación del Consorcio ${ }^{2}$, y el equipo de Pastoral Social ${ }^{3}$, interlocutor del proceso, con quienes se precisaron los objetivos de la sistematización, los núcleos temáticos, el marco conceptual y metodológico, así como, la selección de las ILP y los GSP. Con este equipo se realizaron reuniones de planeación previas al trabajo de campo a los encuentros regionales y al nacional.

La fase de desarrollo comprendió tanto la recolección y análisis de la información. Se continuó la profundización del marco conceptual y metodológico. Se realizó el análisis con base en las categorías determinadas y se desarrolló la escritura de los documentos producto de la sistematización.

Se identificaron las siguientes categorías para estudio: subjetividad, sentidos, prácticas, paz, contexto, intergeneracionalidad e interculturalidad. Fueron referentes importantes en: Subjetividad (Alvarado, Ospina, Botero

2 Universidad Pedagógica, Universidad de Manizales y Fundación Cinde.

3 Conformado por la animadora del área de desarrollo institucional y los asistentes regionales de Pastoral Social. 
\& Muñoz, 2008, Jáidar, 2003, Torres, 2009, Zemelman, 1987, 2004), en Sentidos: (Berger \& Luckman, 1999, González, 2012) en Paz (Lederach, 2007, Muñoz, 2001, Galtung, 1998, 2003, Fisas, 2006) y en Contexto: (Grupo de Memoria Histórica, 2013), entre otros.

El trabajo de campo se realizó a través de encuentros in situ con cada experiencia, el primero a finales del 2013 y el segundo, en el primer semestre de 2014. En el primero, se invitó los líderes a participar en el proceso de sistematización de su ILP. Se llevó a cabo la primera aproximación a la reconstrucción histórica de las experiencias, mediante narraciones de los actores vinculados al proceso; por medio de procedimientos interpretativos (diálogo y contrastación) se realizó la periodización y la línea de tiempo, se identificaron las temáticas que nucleaban el interés de los participantes en la iniciativa, los actores que habían participado en la trayectoria de la experiencia.

Para el análisis de la información se utilizaron procedimientos hermenéuticos basados en el diálogo entre las categorías desarrolladas por los investigadores y las afirmaciones de los sujetos participantes de las ILP y los GSP acerca de sus prácticas y sentidos de paz. Para la "fusión de horizontes" como los llama Gadamer(1997)sedesarrollaron preguntas en relación con la categoría Interculturalidad e intergeneracionalidad, técnica utilizada por la hermenéutica para la interpretación(se interpreta desde unas preguntas afirma Gadamer), se busca hacer una conversación con base en estas y otras que van surgiendo en su transcurso; las preguntas iníciales fueron: ¿Se dan relaciones interculturales e intergeneracionales? ¿Cómo se dan estas relaciones? ¿Qué tipo de relaciones interculturales e intergeneracionales se dan en las experiencias sistematizadas? El horizonte, desde dónde interpretan los investigadores (que como afirma Gadamer pueden ser preconceptos, o unas determinadas tradiciones teóricas) deben ser explicitadas. Con este procedimiento conversacional se buscó develar los sentidos de las prácticas de los sujetos participantes de las experiencias sistematizadas.

En la fase de socialización e incidencia, se realizó el plan respectivo con la definición de los instrumentos y mecanismos para la puesta en común. Además, se realizaron los encuentros regionales y el encuentro nacional.

Hasta el 2015 el SNPS ha construido veinticinco ILP en diferentes partes del territorio nacional, para esta investigación se escogieron ocho experiencias, teniendo en cuenta como criterios los siguientes: que llevaran un tiempo de desarrollo (más de un año); que fueran representativas de trabajar procesos de paz desde diferentes temáticas (estigmatización de poblaciones, reconciliación entre víctimas y victimarios, trabajar por el cuidado de la naturaleza, conflictos interétnicos, ser realizada con niños y jóvenes, producción de alimentos y agricultura orgánica, entre otras); que estuvieran en diferentes regiones del país.

Las experiencias sistematizadas fueron las siguientes:

El Colectivo Sin fronteras apoyado por Ficonpaz ${ }^{4}$ está conformado por un grupo de alumnos $^{5}$ y exalumnos del Colegio Clemencia Holguín de Urdaneta (Institución Educativa oficial de Bogotá), que se ha caracterizado por realizar prácticas formativas, de sensibilización y de participación política, brindando a los y las jóvenes la capacidad de desplegar toda su creatividad y habilidades. Abordan temáticas referidas a: desarrollo de la sexualidad, identidad de género, nuevas formas de relacionarse con los otros y derechos sexuales y reproductivos.

La experiencia Sembradores de paz de La Dorada, desarrolla prácticas relacionadas con la formación de los niños y niñas; orientadas al fortalecimiento de las dimensiones espiritual, personal, social y cultural. El proceso está a cargo de animadoras quienes emplean, diversas herramientas y actividades, algunas retomadas del material pedagógico de Ficonpaz, como las cartillas y otras son propuestas por ellas. Sembradores de Paz promueve los derechos de los niños y potencia el desarrollo de sus capacidades.

La ILP Mujeres Activas, de Facatativá, Cundinamarca, está conformada por mujeres

\footnotetext{
$4 \quad$ Fundación Instituto para la Construcción de la Paz, hace parte de la Arquidiócesis de Bogotá.

5 Para efectos de agilidad en la lectura del texto se emplea el género masculino, aclarando que con ello no se pretende desconocer la perspectiva femenina.
} 
cabeza de familia, trabajadoras de cultivos florales, quienes realizan actividades de formación que buscan fortalecer el autoconcepto, la autoconfianza y la seguridad personal, que les permite reconocer su valía y dignidad como personas. De esta manera, desarrollan la capacidad para enfrentar situaciones de violencia intrafamiliar propias y de otras mujeres de la comunidad. La formación también se desarrolla a nivel técnico, orientada a la elaboración de materiales artísticos y artesanales, como nueva fuente de ingresos, y de fortalecimiento de habilidades sociales.

La experiencia de la ILP de Ventaquemada, Comunidad sin Fronteras, está conformada por habitantes de las veredas Supatá y Puente Piedra. Algunos de sus miembros hacen parte de las juntas de acción comunal. Se organizan como iniciativa después del Paro agrario nacional de agosto de 2013. La práctica que los convoca para fortalecer sus medios de vida es un Proyecto de agricultura orgánica, el que ven como oportunidad para afianzar las relaciones sociales en la comunidad y garantizar la sostenibilidad a futuro del proyecto.

La ILP El Caguán que soñamos (corregimiento de Neiva), está compuesto por un grupo inter étnico (mestizos e indígenas Nasa), realizan reuniones periódicas en las que se hacen talleres sobre el análisis de la situación del corregimiento (sobre el asunto del agua, la salud, la educación, la violencia, la politiquería...). Han realizado talleres para construir la Matriz del futuro deseado. Con esta matriz se plantea un plan de vida para el cumplimiento de los sueños con las dimensiones: personal, comunitaria e institucional. El grupo, entre otras actividades, ha convocado a ollas comunitarias que sirven para acercar y lograr la reconciliación entre grupos de jóvenes de pandillas o entre miembros de la comunidad.

La ILP Recuperación del Río Rumiyaco, en Putumayo, está conformada por una comunidad interesada en recuperar la cuenca de este río, la cual se encuentra deteriorada debido a malas prácticas de los habitantes en su relación con el ecosistema. Han adelantado actividades como: Escuela de líderes, reuniones y caminatas por la cuenca del río Rumiyaco y veredas aledañas, igualmente efectúan mingas, jornadas de limpieza, visitas de evangelización, conversatorios para la recuperación de la historia de la región y gestión con entidades públicas y privadas. También desarrollan talleres sobre ecoturismo, impacto de agroquímicos, alimentación sana, transformación de alimentos, efectos del cambio climático, construcción de huertas y uso de abonos orgánicos.

En la ILP Transformando Relaciones para la Convivencia en el sector de los Comuneros de Garzón Huila, sus miembros participan en retiros, capacitaciones, encuentros de formación con padres de familia, catequesis de bautizo y confirmación. Hay un liderazgo fuerte de las mujeres, que han participado o participan de las juntas de acción comunal. Las actividades de esta ILP han servido para acabar con la estigmatización de los barrios del sector, han logrado reconocimiento en el municipio y han demostrado que pueden celebrar festividades sin violencia. En esta ILP han trabajado personas víctimas de la violencia, señoras desplazadas y excombatientes, han realizado procesos de perdón y olvido.

La ILP Comedor parroquial de Miranda, Cauca, surge de la iniciativa del párroco y un grupo de mujeres vinculadas a la Parroquia para atender a los niños y jóvenes de la comunidad en condición de pobreza y vulnerabilidad social. En esta iniciativa es significativa la gestión para proporcionar el servicio de alimentación a los niños, y garantizarles el derecho a la educación y la salud. Las mujeres de la ILP, realizan gestión con la empresa privada para obtener apoyo en recursos o servicios. La comunidad atiende al llamado del párroco de aportar mercado al comedor en la conciencia de que acogen a un niño más en su familia.

\section{Interculturalidad-Intergeneracionalidad}

Es importante tener en cuenta que las relaciones sociales y culturales no son relaciones idílicas; están construidas en la historia y, por tanto, están atravesadas por relaciones de poder, fuertemente jerarquizadas (Posada-Escobar, Munar-Moreno \& González, 2011).

La investigadora brasileña Vera María Candau(2008)afirmaquevivimosenunasociedad donde se aplican políticas asimilacionistas, que 
reducen las diferencias de los distintos grupos sociales a la cultura hegemónica. Esa posición deslegitima saberes, lenguas, creencias, valores diferentes, pertenecientes a los grupos subordinados, considerados inferiores, explícita o implícitamente.

Candau (2008) considera que es necesaria la interculturalidad, porque esta promueve la relación entre diferentes grupos culturales, presentes en una determinada sociedad. Las ILP y los GSP pueden ser vistos como grupos comunitarios y de niños, niñas, adolescentes y jóvenes NNAJ que promueven la interculturalidad, en ellos se aprende a dialogar las diferencias para desarrollar acciones conjuntas.

Candau piensa que el abordaje intercultural se aproxima al multiculturalismo crítico planteado por Peter McLaren (1997). El multiculturalismo crítico y de resistencia privilegia la transformación de las relaciones sociales, culturales e institucionales en que los significados son generados. Concibe las culturas en continuo proceso de formación, se rehúsa a ver la cultura como no-conflictiva y argumenta que la diferencia debe ser afirmada "dentro de una política de crítica y compromiso con la justicia social" (McLaren, 1997, citado por Candau, 2008, p. 51).

La perspectiva intercultural promueve unas relaciones sociales y culturales basadas en el reconocimiento del otro, para el diálogo entre los diferentes grupos sociales y culturales. Propone la negociación cultural y asumir los conflictos provocados por la asimetría de poder entre los diferentes grupos socioculturales, en nuestras sociedades. La interculturalidad está orientada a la construcción de una sociedad democrática, plural, humana, que articule políticas de igualdad, con políticas de identidad. En las ILP y los GSP se dan procesos de negociación de ideas, se encuentran personas que piensan diferente, que han tenido conflictos y se busca transformar las relaciones entre estas personas, por ejemplo, en la ILP del Caguán, se reúnen personas que históricamente no se reconocían, se despreciaban, en la ILP de Garzón participan víctimas del conflicto armado y ex miembros de los grupos armados.

Tal como lo afirma Lederach en entrevista para la revista semana.com en la sección Nación:
La paz no es un acuerdo firmado en Cuba entre el gobierno colombiano y las FARC. La paz se logra cuando cada colombiano asuma el respeto por la diferencia y establezca relaciones constructivas con el otro, con ese otro al que durante más de medio siglo no ha querido o no ha podido escuchar (Lederach, 2015).

La interculturalidad supone la creación de espacios sociales para el intercambio entre personas, conocimientos, saberes y prácticas culturalmente diferentes, buscando desarrollar un nuevo sentido entre ellas, en su diferencia. Un espacio de negociación y de traducción, donde las desigualdades sociales, económicas y políticas, las relaciones y los conflictos de poder de la sociedad, no son mantenidos ocultos, sino reconocidos y confrontados (Posada-Escobar, Munar-Moreno \& González, 2011).

Para Fleuri, la noción de interculturalidad tiene carácter propositivo y político, convirtiéndose en una categoría central para diferentes propuestas sociales y educativas. La noción de interculturalidad, además de proponer condiciones para el fortalecimiento de la identidad, también estimula la adquisición de conocimiento cultural de otros pueblos, de los diferentes grupos sociales y de otras culturas. El SNPS con su metodología de construcción de paz, no sólo ha propiciado el encuentro entre las personas de una misma comunidad, sino también el tejido de relaciones con otros grupos comunitarios que realizan procesos de transformación social, de ahí la importancia de los Encuentros regionales y nacionales.

De otra parte, las investigadoras Corsi y Freitas (2010) consideran que es posible "cuestionar valores, estereotipos y actitudes llenas de prejuicios, vistas, muchas veces, como naturales en nuestra sociedad, y es necesario la agencia en la construcción de una sociedad democrática" (p. 159). Las ILP y los GSP han trabajado para romper los estereotipos establecidos sobre diferentes poblaciones $\mathrm{y}$ grupos sociales; en varias experiencias son notorias las acciones para lograr que no se estigmatice a los jóvenes como "consumidores", para hacer que determinados barrios no sean vistos como espacios "peligrosos". En el caso del Colectivo Sin Fronteras, quienes realizan 
acciones que resaltan la dignidad de los jóvenes con diferentes orientaciones sexuales.

Todas estas prácticas han gestado el encuentro y la convivencia en medio de la diferencia generacional, tanto las ILP como los GSP se han venido consolidando como espacios de construcción intergeneracional al convocar a NNAJ a sus actividades, por ejemplo, en la ILP Mujeres Activas, son los estudiantes de servicio social quienes han reactivado la participación del grupo en un momento de crisis; en la experiencia del Colectivo Sin Fronteras los jóvenes han empezado a permear la subjetividad de sus maestros, padres y vecinos del sector con sus puestas en escena y activismos concientizadores ${ }^{6}$.

En coherencia con estas prácticas, Fleuri (2006) plasma una visión intercultural de las relaciones generacionales, él considera que, en cuanto a la infancia, es necesario el reconocimiento del otro a partir de los complejos procesos que constituyen su subjetividad, para comprenderla:

...es preciso ver y oír a los niños, a partir de sí mismos, en su alteridad y positividad, como sujetos productores de cultura... No se trata de reducir al otro a lo que nosotros pensamos o queremos de él. No se trata de asimilarlo a nosotros mismos, excluyendo su diferencia (Fleuri, 2006, p. 70).

Según sus postulados, hay que estar atentos para acoger lo que los niños y las niñas dicen en su alteridad, para comprender los sentidos que sus acciones y posiciones asumen en el contexto de sus respectivos patrones culturales. La adopción de una imagen universal de niño, niña y de infancia sería productora de desconexión con los hechos y acontecimientos que envuelven sus vidas, en la cotidianidad. Este enfoque propone la necesidad de construir escenarios para la negociación cultural, que enfrente los conflictos provocados por la asimetría de poder entre los diferentes grupos socioculturales, en nuestras sociedades.

6 Expresión empleada por el colectivo Sin Fronteras para referirse a un conjunto de acciones que incluyen performances, representaciones, música, expresión y pintura corporal, entre otras.
El concepto de interculturalidad propone fortalecer la identidad, pero también promueve el intercambio, la relación con las otras culturas. Para entender las relaciones interculturalesintergeneracionales, se requiere adoptar otras perspectivas epistemológicas que piensen las identidades no de manera esencialista, inamovible, sino como formas de pensar que consideren las interrelaciones, el hibridismo y la ambivalencia.

En las relaciones intergeneracionales hay todo un cúmulo de posibilidades, conflictos y transformaciones culturales, así como una serie de procesos importantes que se dan en las relaciones interculturales. En estas interrelaciones o "entre lugares" como los denomina Bhabha, (1998 citado por Fleuri, 2006,p. 9) se dan los procesos de subjetivación y de constitución de identidades. De ahí también, que estas identidades sean móviles y estén cruzadas por aspectos de la realidad étnicos, raciales, lingüísticos, religiosos, nacionales $\mathrm{y}$ generacionales.

Cuando se analizan las relaciones intergeneracionales muchas veces se cree que son sólo de sometimiento, de dominación, pero para Fleuri (2006) se dan múltiples interrelaciones, interdependencias, hibridismos, e influencias mutuas.

Por esto se requieren estudios y análisis de los sujetos en sus contextos sociales y en la forma como estos sujetos construyen sus identidades. Fleuri (2006) presenta varias investigaciones en las que se estudian los conflictos entre jóvenes y profesores, y plantea la necesidad de desarrollar estrategias interculturales para tramitar dichos conflictos, estrategias basadas en "la interacción y la acogida de lo diferente".

Cuando sehabla de un enfoque interculturalintergeneracional para analizar las prácticas de paz de las ILP y de los GSP, se puede preguntar, entonces: ¿Cómo estas pueden ser espacios de acogida de la diferencia? ¿Cómo promueven la participación de los niño/as y jóvenes? ¿Cómo se trabajan, se piensan y se tramitan las diferentes formas en que los sujetos actúan, habitan en los barrios y en los territorios?

La significación que se tiene de la infancia y la juventud, lleva a realizar intervenciones $\mathrm{o}$ interacciones desde visiones adultocéntricas 
acerca de ellos, una visión centrada en los déficits supone unas acciones y no permite otras. Realizar un trabajo interculturalintergeneracional para promover la paz supone entonces, abrir los espacios de las organizaciones a la participación de los niños/ as y jóvenes, pero también, analizar las ideas que se tienen sobre ellos-ellas, reconocer las pautas culturales, para reconstruirlas.

Estas ideas sobre las relaciones intergeneracionales son claves para interpretar y orientar las prácticas en los procesos de paz que se construyen en las ILP y los GSP. Prácticas donde las historias de vida, los saberes de los jóvenes son reconocidas y donde estos luchan por romper los estereotipos y las estigmatizaciones como "jóvenes problema".

\section{Intergeneracionalidad como Apoyo y Solidaridad}

Diferentes estudios muestran la importancia de los programas Intergeneracionales (HattanYeo \& Ohsako, 2001, Sánchez et al., 2007, Sánchez \& Díaz, 2005), en especial en los países donde la población se ha envejecido. La idea central que acompaña estos programas es que: "La intergeneracionalidad tiene que ver con la interdependencia, con la solidaridad y con la reciprocidad $\mathrm{y}$, por tanto, no consiste únicamente en que las generaciones estén juntas" (Mariano et al., 2007, p. 34).

Los Programas Intergeneracionales se han desarrollado por medio de políticas públicas en diferentes países y con el respaldo de organismos internacionales, actualmente se impulsan de manera intencionada y en este sentido se han convertido en un campo de estudio.

En complemento de lo anterior Jesús García (2003) plantea que se asiste a un "interés nuevo" por los temas relacionados con la intergeneracionalidad. El autor señala la importancia de la educación intergeneracional (EI) y la define como: "un diálogo (1) libre (2) entre personas de culturas distintas que, partiendo de campos y motivaciones comunes (3), intenta descubrir los valores entre iguales (4) para enriquecer los proyectos de vida de las personas" (p. 20).
Así mismo, García afirma que la EI actúa en diferentes ámbitos o espacios en los que esta se puede realizar: el creativo, el ecológico, el lúdico y el de compromiso social. Esta idea de los ámbitos es muy pertinente para la presente investigación, puesto que permite ver y proponer cómo las generaciones se relacionan, intercambian no sólo porque participen del mismo proceso organizativo, sino que estas generaciones se encuentran, se educan y aprenden conjuntamente cuando se abren espacios artísticos culturales, cuando se generan momentos para la recreación, el deporte, la festividad, la celebración, el cuidado del ambiente y en la actividad de solidaridad social.

Para Aura Márquez (s. f.), que ha realizado diferentes investigaciones sobre el papel de los abuelos y las abuelas, las relaciones intergeneracionales son variadas y dependen de diferentes factores como la edad, las relaciones de género y la clase social, entre otras.

Según Márquez, para pensar las relaciones intergeneracionales, es necesario tener en cuenta que los viejos y las viejas son personas con una visión del tiempo diferente a la de los otros grupos de edad, por la experiencia y "con una visión hacia el futuro distinta, que en muchos casos es un 'no futuro', por las condiciones de salud, económicas y sociales existentes" (Márquez, s. f., p. 5).

Márquez retoma los planteamientos de Margaret Mead (1990) sobre las posibilidades de rupturas generacionales. En las relaciones posfigurativas, los adultos pueden transmitir sus valores a sus hijos, los/las abuelos- abuelas piensan que sus nietos van a seguir sus pasos. En las relaciones configurativas los hijos y nietos asumen la cultura de sus padres/madres y abuelo/as, pero las nuevas generaciones cambian de acuerdo a los cambios sociales de la época. En las relaciones prefigurativas, las generaciones jóvenes orientan sus vidas sin tener en cuenta las generaciones anteriores, dándose una ruptura generacional.

Estas formas diversas de relación intergeneracional se expresan en las ILP y los GSP. En la mayoría de los miembros de las ILP prima la postura posfigurativa que determina lo que es deseable para el entorno en que se 
desenvuelven los NNAJ, así como sus valores, sus formas de ser y comportarse, en coherencia con unos principios y unas creencias de orden social y religioso. Es notorio el interés en favorecer entornos seguros, para proteger a los NNAJ de las sustancias psicoactivas y de las situaciones de riesgo a que pueden exponerse. En contraste, en el Colectivo Sin Fronteras, se evidencia la tensión entre las relaciones posfigurativas establecidas por los adultos y las prefigurativas que proponen los NNAJ.

\section{La Estrategia de Transformación Social del SNPS como dispositivo para la construcción de subjetividades políticas de Paz}

El dispositivo supone un conjunto de elementos articulados y constituye toda una serie de mecanismos que configuran las subjetivaciones, que contribuyen a formar un tipo de sujeto.

El concepto de dispositivo incluye elementos variados: está conformado por una serie de estrategias metodológicas y también de discursos, de disposiciones y formas de organizar los espacios, los tiempos en un proceso educativo.

El educador español Jorge Larrosa (1995) ha desarrollado y aplicado el concepto de dispositivo para el análisis de las prácticas pedagógicas y sus diferentes metodologías:

Un dispositivo pedagógico será, entonces, cualquier lugar en el que se constituye o se transforma la experiencia de sí. Cualquier lugar en el que se aprenden o se modifican las relaciones que el sujeto establece consigo mismo. Por ejemplo, una práctica pedagógica de educación moral, una asamblea en un colegio, una sesión de un grupo de terapia, lo que ocurre en un confesionario, en un grupo político, o en una comunidad religiosa, siempre que esté orientado a la constitución o a la transformación de la manera en que la gente se describe, se narra, se juzga o se controla a sí misma (Larrosa, 1995, p. 291).

Larrosa (1995) analiza los dispositivos de autogobierno, relacionados con el ver, decir o expresar, juzgarse, narrarse y hacer. Son mecanismos pedagógicos muy utilizados en la educación. Esto se puede relacionar con las propuestas pedagógicas que en la iglesia católica se han configurado en tres elementos metodológicos el ver, juzgar y actuar.

En síntesis, los dispositivos los podemos entender como un conjunto de elementos enlazados que contribuyen a formar unas determinadas subjetividades, por esto se considera que la estrategia de intervención propuesta por el SNPS para construir procesos de paz, se constituye en un dispositivo que permite construir unas subjetividades políticas de paz.

La propuesta de intervención del SNPS puede ser vista como un dispositivo para la transformación social y para la construcción de subjetividades políticas de paz. Por esto, es necesario explicitar dicha estrategia porque gran parte de las actividades y los sentidos de paz que se encuentran en las ILP y los GSP están orientadas por ella.

La estrategia de intervención (SNPS, s. f.) propone cuatro niveles de transformación: personal, relacional-comunitario, organizacional y estructural. Este proceso incluye varias etapas: la respuesta solidaria en momentos de crisis (como el desplazamiento forzado de la población), reconstrucción del tejido social, el diseño del futuro deseado, la realización de planes y acciones para acercar el futuro.

El Futuro deseado es un elemento central de la estrategia de intervención, consiste en acompañar y animar a las comunidades para que definan y construyan de manera participativa "el futuro deseado"; pensado como "el goce efectivo de derechos y el cumplimiento de deberes..."

Entre los componentes de la estrategia de intervención están: formación, investigación, incidencia política, intervención psicosocial, atención humanitaria, atención jurídica y comunicación (SNPS, s. f., p. 34).

El SNPS hace un énfasis en la reflexión pedagógica, en torno a las relaciones que se crean entre las personas, sobre el saber de las comunidades y en las transformaciones que se logran con estos procesos formativos que buscan construir Comunidades de Aprendizaje. 
Para implementar en la práctica la estrategia de intervención, el SNPS conforma los equipos u organismos levadura (EL u OL), que se constituyen en los núcleos organizativos que dinamizan las ILP, compuestos por líderes y otros actores claves de las comunidades.

En síntesis, se puede afirmar que la estrategia de intervención de SNPS se constituye en un dispositivo pedagógico, compuesto por un conjunto de acciones, estrategias, disposiciones, discursos que ayudan a construir unas subjetividades políticas de paz.

\section{Lo intercultural y lo intergeneracional en las ILP y los GSP}

Tomando en consideración que el análisis de las experiencias de las ILPS y los GSP desde una perspectivainterculturale intergeneracional, fue uno de los propósitos más relevantes en esta investigación, presentamos los principales hallazgos, estableciendo las características de esta perspectiva en las experiencias.

La intergeneracionalidad supone la interacción constante entre generaciones coexistentes, tal es el caso de algunas de las experiencias promovidas por el SNPS para la construcción de Paz, en donde se vinculan varias generaciones de NNAJ y adultos interactuando desde su mirada del mundo para consolidar y/o constituir propuestas de paz. Por lo tanto, estas experiencias son pequeños enjambres de grupos intergeneracionales ligados por la cotidianidad compartida y la búsqueda de un sueño o futuro deseado.

En esta sistematización se ha podido constatar diferentes tipos de relaciones intergeneracionales: por un lado, hay una preocupación permanente de las personas adultas por los NNAJ de sus comunidades ante los peligros que los acechan (consumo de sustancias psicoactivas, ser reclutados por pandillas o grupos armados) y esto, los lleva a realizar acciones de prevención como la creación de grupos culturales (danzas, música), creación de campeonatos y equipos deportivos. Es decir, toda una serie de acciones de protección, de cuidado, dirigidas hacia los NNAJ, sin que se fomente su protagonismo, de manera que este grupo generacional es objeto de su acción, sin generar autonomía, es decir, esta población es "asistida". Se trata entonces, de un primer nivel de relacionamiento que por supuesto requiere una reflexión sobre el lugar de los NNAJ en las comunidades, sus posibilidades de participación y la oportunidad de generar un intercambio reciproco en la construcción de acciones para la paz.

De otra parte, la experiencia de Sembradores de Paz, del Colectivo Sin Fronteras es visible por su autonomía, lo que representa mayor dificultad y conflicto en las relaciones con la autoridad de la institución educativa. La expresión creativa desarrollada por este grupo no es tan fácilmente aceptada por las personas adultas, ellas quisieran que las formas y los contenidos de expresión estuvieran orientadas por ellos, que se realicen conforme a sus lineamientos y tiempos (Encuentro in situ, 2014). Sigue presente la necesidad de andamiaje para llevar esta relación intergeneracional a un nivel reciproco en que se contemple el lugar del otro desde una mirada colaborativa y articulada.

En el marco de estas tensiones, las ILP se construyen como espacios de interlocución, donde se pueden expresar las diferencias, espacios donde se manifiestan creencias e ideas y se desarrollan acciones e iniciativas de manera colaborativa. A su vez, los encuentros regionales y nacionales, son espacios más amplios de participación e intercambio de experiencias que potencian la capacidad de incidencia política. En este aspecto encontramos un nivel de relación que facilita el encuentro de las diferencias (generacionales, ideológicas políticas, de género) y las pone en diálogo para propiciar agenciamiento colectivo, se trata del ejercicio de una interculturalidad critica que permite hacer conciencia de que se puede construir para el bien común en el marco de disensos.

En esta línea de reflexión se encuentra la ILP el Caguán que soñamos, indígenas y campesinos con tradiciones e intereses particulares, quienes en medio de contradicciones y conflictos, logran debatir y encontrar articulaciones para el uso común de la tierra y el agua, aun cuando no existan consensos plenos sobre los aspectos que debaten (Encuentro in situ, 2014). 
Esta idea de una interculturalidad crítica toma nuevos matices en otras experiencias de las ILP, como en la de Garzón, en donde se ha trabajado la construcción de paz tratando de quebrar la estigmatización hacia los habitantes de unos barrios del municipio y la exclusión que hay sobre los/as jóvenes señalados como adictos.

Las personas de las ILP piensan que la paz se construye desde la familia al reconocer sus problemáticas internas y generar relaciones sanas entre sus miembros. La mirada desde la perspectiva intercultural - intergeneracional permite explorar las transformaciones que en tiempos contemporáneos viven los sujetos, nuevas formas de familia, de escuela, nuevas lógicas de organización, movimientos que agrupan las demandas e inquietudes de la población, afectan su mirada sobre la paz y vinculan elementos propios del ser y estar juntos en un territorio.

En las ILP podemos constatar que las mamás y las abuelas/os como en muchos lugares del país, cuidan los hijos y nietos para que las hijas e hijos puedan trabajar. Adicionalmente, las mujeres de las ILP han realizado esfuerzos importantes para que el Estado atienda a los niño/as en jardines infantiles y comedores. En la Iniciativa de Miranda, han logrado, en acción conjunta con el párroco, la asignación de una partida del presupuesto municipal para el sostenimiento del Comedor parroquial, así como, el acceso a servicios de salud y educación, en el marco de garantizar como sociedad estos derechos a los niños y niñas vinculados a la ILP. La dinámica de funcionamiento del Comedor se convierte en un dispositivo de socialización, donde se promueve el respeto, el reconocimiento de cada quien como diferente y único, con potencialidades a desarrollar.

En sintonía con la lectura del contexto y de los cambios fundamentales en las formas de organización, emergen en las experiencias características propias de la perspectiva intercultural-intergeneracional que tienen que ver con el tratamiento directo de las diferencias, las formas alternativas de tramitarlas y de generar acciones que incidan en las dinámicas de construcción de paz.

Rev.latinoam.cienc.soc.niñez juv 15 (1): 505-517, 2017

http://revistalatinoamericanaumanizales.cinde.org.co

DOI:10.11600/1692715x.1513222042016
Al respecto, la participación de las mujeres Activas de la ILP de Facatativá, en espacios de formación para prevención de la violencia de género ofrecidos tanto por la Iglesia como por la Secretaría de Desarrollo social del municipio, les ha permitido realizar una labor de sensibilización y orientación sobre este tópico en las relaciones familiares y de la comunidad focal.

El profesor Isidro del Equipo levadura de Garzón ha aprovechado toda su experiencia comunitaria y su participación en la ILP para enriquecer su trabajo en la escuela. Él reflexiona sobre:

¿Cómo se hace la inclusión? ¿Cómo se logra no marginalizar, no invisibilizar al otro? (Entrevista al profesor Isidro, 2014, p. 12).

Según el profesor Isidro se requiere de una serie de estrategias (para el caso de la investigación, elementos que conformen un dispositivo) para incluir, dar voz y liderazgo a los jóvenes:

Mire la líder, la niña que está ahorita de vocera, ella es desplazada, era agresiva, peleona, me le daba a todo el mundo duro, ahora es líder, entonces esa transformación que uno va haciendo con los niños poco a poco, pero haciéndolo desde irles dando liderazgos, a ella le gustaba bailar y no la dejaban bailar porque era pobre, entonces trabajé para que la dejaran bailar.

La mamá la tiene en la cárcel, vienen por allá de San Vicente del Caguán, por allá de la "quinta porra", al papá lo mataron. Entonces, vienen de una crisis terrible, entonces la niña se sienta con uno a contarle todas esas historias, entonces yo hago que ella escriba, ella está comenzando ya a escribir, a escribir sus historias. Y ese mismo trabajo lo hemos hecho con los adultos reinsertados y desplazados, ellos también contaban sus historias (Entrevista al profesor Isidro, 2014, p. 12).

De acuerdo con lo expuesto se puede afirmar que en la estrategia de intervención del SNPS, existen elementos y relaciones que potencian un dispositivo intercultural e intergeneracional. Y si bien, en el Encuentro 
de la regional Suroccidente se hicieron visibles nuevas ILP constituidas por adolescentes y jóvenes, se ha podido apreciar a través de la sistematización de las experiencias, cómo lo intergeneracional y lo intercultural se manifiestan de diversas formas enriqueciendo el dispositivo. Para definir y brindar alternativas de profundización, es importante interrogarse acerca de ¿Cómo los discursos y las prácticas interculturales e intergeneracionales pueden integrarse a la estrategia de intervención para la transformación social? ¿De qué manera el trámite de las diferencias intergeneracionales e interculturales aporta a la emergencia de nuevas subjetividades de paz?

Igualmente, vale la pena cuestionarse acerca de ¿Qué tanto espacio y oportunidad de participación tienen los/las jóvenes en las organizaciones? ¿Qué tanto atraen a los/ as jóvenes este tipo de organizaciones? ¿Qué propuestas pueden entretejer las ILP al respecto? ¿Cómo aportan las ILP a procesos de paz posibilitando romper los estereotipos sobre los niño/as, por ejemplo: como seres inferiores, sin capacidad de decidir, participar y sin voz; o sobre lo juvenil como lo irrespetuoso, peligroso e improductivo?

\section{Lista de referencias}

Alvarado, S. V., Ospina, H. F., Botero, P. \& Muñoz, G. (2008). Las tramas de la subjetividad política y los desafíos a la formación ciudadana en jóvenes. Revista Argentina de Sociología, 6 (11), pp. 19-43.

Berger, P. \& Luckman, T. (1999). La construcción social de la realidad. Buenos Aires: Amorrortu.

Candau, V. (2008). Derechos humanos, educación e interculturalidad: Las tensiones entre igualdad y diferencia. Revista Brasileira de Educação, 37 (13), pp. 45-56. Recuperado de:

h t t p://ww w. s c i elo.br/scielo. php? script $=$ sci arttext $\&$ pid $=\mathrm{S} 1413$ $24782008000100005 \& \operatorname{lng}=$ pt\&nrm $=$ iso $\&$ tlng $=\mathrm{pt}$

Cendales, D., Mariño, G. \& Posada, J. (2004). Aprendiendo a sistematizar. Bogotá, D. C.: Cosude.
Corsi, A. \& Freitas, E. (2010). Práticas pedagógicas no ensino fundamental na perspectiva do multiculturalismo crítico. Currículo sem Fronteiras, 2 (10), pp. 158-182. Recuperado de: http://www.curriculosemfronteiras.org/ vol10iss2articles/corsi-lima.htm

Fisas, V. (2006). Cultura de paz y gestión de conflictos. Barcelona: Unesco.

Fleuri, R. (2006). Intercultura y Educación. Revista Virtual Astrolabio, 4. Recuperado de:

http://www.astrolabio.unc.edu.ar/articulos/ multiculturalismo/articulos/fleuri.php

Gadamer, H. (1997). Verdad y Método. Salamanca: Sígueme.

Galtung, J. (1998). Tras la violencia. $3 R$ : reconstrucción, reconciliación, resolución. Afrontando los efectos visibles e invisibles de la guerra y la violencia. Bilbao: Bakeaz.

Galtung, J. (2003). Paz por medios pacificos. Paz y conflicto, desarrollo y civilización. Bilbao: Gernika Gogoratuz.

García, J. (2003). La Educación Intergeneracional. Un Nuevo Ámbito Educativo. Madrid: Dykison.

González, F. (2012). La subjetividad y su significación para el estudio de los procesos políticos: Sujeto, sociedad y política. En C. Piedrahíta-Echandía, Á. Díaz-Gómez, P. Vommaro (comps.) Subjetividades politicas: Desafios y debates latinoamericanos, (pp. 5-11). Bogotá, D. C.: Universidad Distrital Francisco José de Caldas, Clacso.

Grupo de Memoria Histórica (2013). ¡Basta ya! Colombia: Memorias de guerra y dignidad. Informe General Grupo de Memoria Histórica. Bogotá, D. C.: Centro Nacional de Memoria Histórica.

Hattan-Yeo, A. \& Ohsako, T. (2001). Programas intergeneracionales: Política pública e implicaciones de la investigación. Una perspectiva internacional. Hamburgo: Instituto de Educación de la Unesco y The Beth Johnson Foundation. Recuperado de: http://www.unesco.org/education/uie/pdf/ intergenspa.pdf 
Jáidar, I. (2003). Tras las huellas de la subjetividad. México, D. F.: UAM.

Larrosa, J. (1995). Escuela, poder y subjetivación. Colección Genealogía del poder. Madrid: La Piqueta.

Lederach, J. (2007). La imaginación moral: El arte y el alma de la construcción de la paz. Bilbao: Bakeaz, Gernika Gogoratuz.

Lederach, J. (2015). La paz la construye cada colombiano. Entrevista con John Paul Lederach. Recuperado de: http://www. semana.com/nacion/articulo/la-firmaabrira-heridas/452307-3

Márquez, A. (s. f.) Una mirada a las relaciones intergeneracionales en la familia desde la vejez. Recuperado de: http://www.bdigital. unal.edu.co/1363/27/26CAPI25.pdf

Mead, M. (1990). Cultura y Compromiso, estudio sobre la ruptura generacional. México, D. F.: Gedisa.

Muñoz, F. A. (ed.) (2001). La paz imperfecta. Granada: Universidad de Granada.

Pastoral Social-Cáritas Colombia (2015). Quiénes somos. Bogotá, D. C: Pastorial Social-Cáritas Colombia. Recuperado de: http://www.new.pastoralsocial.org/ conocenos/quienes-somos/historia-snps

Posada-Escobar, J. J., Munar-Moreno, Y. A. \& González, M. (2011). La interculturalidad y el diálogo de saberes en la formación de educadores de las escuelas normales para la atención educativa a poblaciones. Bogotá, D. C.: Ciup, UPN.

Ramírez, J. (2009). Producción de conocimientos pedagógicos desde y sobre las experiencias educativas. Lectura introductoria del Módulo: Sistematización de Experiencias. Posibilidad de producción de conocimientos teórico-prácticos. Bogotá, D. C.: Maestría en Desarrollo Educativo y Social, Cinde, Universidad Pedagógica Nacional.

Sánchez, M. \& Díaz, P. (2005). Los Programas Intergeneracionales. En S. Pinazo \& M. Sánchez(eds.) Gerontología, actualización, innovación y propuestas, (pp. 393-430). Madrid: Pearson, Prentice Hall.

Sánchez, M., Butts, D., Hatton-Yeo, A., Henkin, N., Jarrot, S., Kaplan, M. (...) Weintraub, A. (2007). Programas Intergeneracionales.
Hacia una sociedad para todas las edades. Barcelona: Colección Estudios Sociales 23, Fundación La Caixa. Recuperado de: http://www.aepumayores.org/sites/default/ files/Programas_Intergeneracionales Coleccion_Estudios_Sociales_vo123_es.pdf.

Secretariado Nacional de Pastoral Social (s. f.) Estrategia de Intervención del Secretariado Nacional de Pastoral Social. Bogotá, D. C.: Secretariado Nacional de Pastoral Social.

Torres, A. (2009). Acción colectiva y subjetividad. Un balance desde los estudios sociales. Folios, (30), pp. 51-74.

Zemelman, H. (1987). Conocimiento y sujetos sociales. Contribución al estudio del presente. México, D. F.: El Colegio de México.

Zemelman, H. (2004). En torno de la potenciación del sujeto como constructor de historia. En M. C. Laverde, G. Daza \& M. Zuleta (eds.) Debates sobre el sujeto. Perspectivas contemporáneas, (pp. 91-97). Bogotá, D. C.: Universidad Central, Diuc, Siglo del Hombre Editores. 